\title{
The Physics of Transient High-Energy Cosmic Sources: from Radio to VHE Gamma-Rays
}

\section{Meintjes P.J.*}

Department of Physics, University of the Free State, PO Box 339, Bloemfontein, 9300, South Africa

E-mail: MeintjpJ@ufs.ac.za / MeintPJ@gmail.com

In this study magnetohydrodynamical processes that may drive particle acceleration and transient emission in the cataclysmic variables, especially the enigmatic transient multi-frequency nova-like variable, AE Aquarii, are reviewed. It is shown that the propeller ejection of material from the magnetosphere of the white dwarf may drive magnetospheric processes that can accelerate charged particles to high energies. For example, the propeller ejection of the mass transferred from the secondary star results in magnetic reconnection as well as the induction of huge field aligned potentials that may accelerate charged particles to very high energies (VHE) $\varepsilon>10^{11} \mathrm{eV}$. These processes have been discussed to provide a theoretical framework to evaluate the non-thermal emission detected from this source, especially the transient burst-like VHE emission reported by two independent groups in the 1990s. In conclusion very preliminary results have been presented of a search for pulsed emission from AE Aquarii in the Fermi-LAT data.

XI Multifrequency Behaviour of High Energy Cosmic Sources Workshop,

25-30 May 2015

Palermo, Italy

\footnotetext{
* Speaker.
} 


\section{Introduction}

It is probably fair to say that the driving force, or catalyst, behind most transient or dynamic phenomenona in astrophysical environments is related to magnetic fields. Most of the universe is in the form of plasma threaded by magnetic fields. Here one may think invariably of the role magnetic fields play in the plasma dynamics of strongly magnetized white dwarfs in the magnetic cataclysmic variables (e.g. [1]), as well as disc accreting compact objects in X-ray binaries (e.g. [2]), and also in turbulent accretion discs to provide the required magnetic viscosity that can drive the observed mass accretion rates (e.g [3, 4]). It has been shown that magnetic fields play a fundamental role in the magnetospheric propellering in disc accreting neutron star systems (e.g. [5]), as well as the cataclysmic variables, for example the enigmatic nova-like variable AE Aquarii (e.g. [6, 7, 8, 9, 10]).

The detection of radio emission from the magnetic cataclysmic variable AM Her sparked huge interest in these sources being potential non-thermal radio sources [11, 12]. These authors suggested that a potential lack of synchronization between the magnetized white dwarf and the secondary mass donating star may induce strong field aligned potentials that can accelerate electrons resulting in synchrotron emission at, among others, radio wavelengths. Non-thermal radio outbursts have been detected from the nova-like variable system AE Aquarii [13] and quantified in terms of expanding synchrotron emitting clouds [14]. This detection was the first clear indication that particle acceleration can occur in a cataclysmic variable. Subsequently, radio emission was detected from non-magnetic and magnetic cataclysmic variables (e.g [15]). It was found that the level of radio emission was related to the asynchronisity of white dwarf rotation with respect to the binary period. The culmination of this effect must be the white dwarf in AE Aquarii, which is probably among the most asynchronous rotators of all cataclysmic variables. Reports of Very High Energy (VHE) Gamma-ray emission $\left(\varepsilon_{\gamma} \geq 10^{11} \mathrm{eV}\right)$ from the novalike variable AE Aquarii have been made by two independent groups (e.g $[16,17,18])$ in the 1990s. Unfortunately these could not be verified by more recent campaigns (e.g. [19, 20,21]). These examples, by far not comprehensive, provide evidence of the fascinating MHD processes driving particle acceleration and non-thermal radio outbursts and possibly VHE gamma-ray emission in close binary systems like the cataclysmic variables.

In this study I am going to focus on the processes that may drive the multi-wavelength transient emission in the enigmatic nova-like variable system AE Aquarii. The paper will be structured as follows: In the following section a general discussion will be presented of relevant particle acceleration processes applicable to AE Aquarii, which will be followed by a discussion of the reported VHE emission in AE Aquarii.

\section{Particle Acceleration}

Apart from influencing bulk flow kinematics of a plasma, magnetic fields also play a fundamental role in particle acceleration processes [22] and subsequent non-thermal emission in astrophysical environments. It can be shown (e.g. [23]) that electric and magnetic fields in a reference 
frame co-moving with a highly conducting fluid or plasma can be expressed as

$$
\begin{aligned}
\mathbf{E}^{\prime} & =\Gamma\left(\mathbf{E}+\frac{1}{c}(\mathbf{v} \times \mathbf{B})\right) \\
\mathbf{B}^{\prime} & =\Gamma\left(\mathbf{B}-\frac{1}{c}(\mathbf{v} \times \mathbf{E})\right),
\end{aligned}
$$

where $\left(\mathbf{E}^{\prime} ; \mathbf{B}^{\prime}\right),(\mathbf{E} ; \mathbf{B})$ represent the fields in the co-moving and laboratory frames respectively and where $\Gamma$ represents the bulk Lorentz factor. Here $\mathbf{v}$ represents the relative velocity between the fluid (co-moving) frame and the stationary (laboratory) frame. For a highly conducting fluid (i.e. $\sigma \rightarrow \infty$ ), the electric field in the co-moving frame $\mathbf{E}^{\prime} \rightarrow \mathbf{0}$ and field quantities transform as follows

$$
\begin{aligned}
& \mathbf{E}=-\Gamma\left(\beta \times \mathbf{B}^{\prime}\right) \\
& \mathbf{B}=\Gamma \mathbf{B}^{\prime}
\end{aligned}
$$

where $\beta=\frac{\mathbf{v}}{c}$. Notice that the electric field responsible for the acceleration of individual charged particles is tied to the bulk flow velocity $(\mathbf{v})$ of the magnetized fluid with respect to the laboratory (stationary) reference frame. The maximum energy that can be attained by a charged particle can be determined by

$$
\begin{aligned}
\varepsilon_{\max } & =Z e \int \mathbf{E} . \mathbf{d s} \\
& =q \beta \Gamma R_{\mathrm{s}} B^{\prime}
\end{aligned}
$$

where $Z e$ is the charge and $\beta=(v / c), \Gamma$ represent the velocity and Lorentz factor. In a fast rotating magnetosphere of a compact object like a neutron star or white dwarf, these parameters are associated with the rotational velocity of the source with respect to a stationary observer. Here $R_{\mathrm{S}}$ and $B^{\prime}$ represent the size of the source and the magnetic field in the frame of the source respectively. This expression clearly illustrates that for fast rotating compact objects, satisfying $(\beta \Gamma>>1)$, the rotational kinetic energy reservoir relaxes the requirements for the strength of the intrinsic magnetic fields in the source to accelerate charged particles to high energies. It can be seen from Figure 1 that even white dwarfs, with magnetic fields ranging between $\sim 10^{5} \mathrm{G}-10^{8} \mathrm{G}$, can accelerate charged particles to energies of the order of $\varepsilon_{\max } \sim 10^{20} \mathrm{eV}$ [24].

The equation above plays a fundamental role to gauge the potential of rotating magnetized compact objects to accelerate charged particles to very high energies (e.g. [25]). This essentially means that the electric field is in fact just the induced emf as long as there is relative motion between the co-moving (source) frame and the observer. A similar expression can be derived in the non-relativistic limit, by utilizing the magnetic induction equation [22], i.e.

$$
\frac{\partial B}{\partial t}=\nabla \times \mathbf{v} \times \mathbf{B}-\nabla \times \eta \nabla \times \mathbf{B},
$$

where $\eta=\frac{c^{2}}{4 \pi \sigma}$ represents the resistivity of the plasma. This leads to two field components, perpendicular and parallel to the magnetic fields, i.e.

$$
\begin{aligned}
\mathbf{E}_{\perp} & =-\frac{\mathbf{v}}{c} \times \mathbf{B}\left[1+\left(\frac{1}{R_{\mathrm{m}}}\right)\left(\frac{L v}{c}\right)\left(\frac{\nabla \times \mathbf{B}}{\mathbf{B} \times \mathbf{v} / c}\right)\right] \\
\mathbf{E}_{\|} & =\left(\frac{1}{R_{\mathrm{m}}}\right) \mathbf{E}_{\perp} .
\end{aligned}
$$




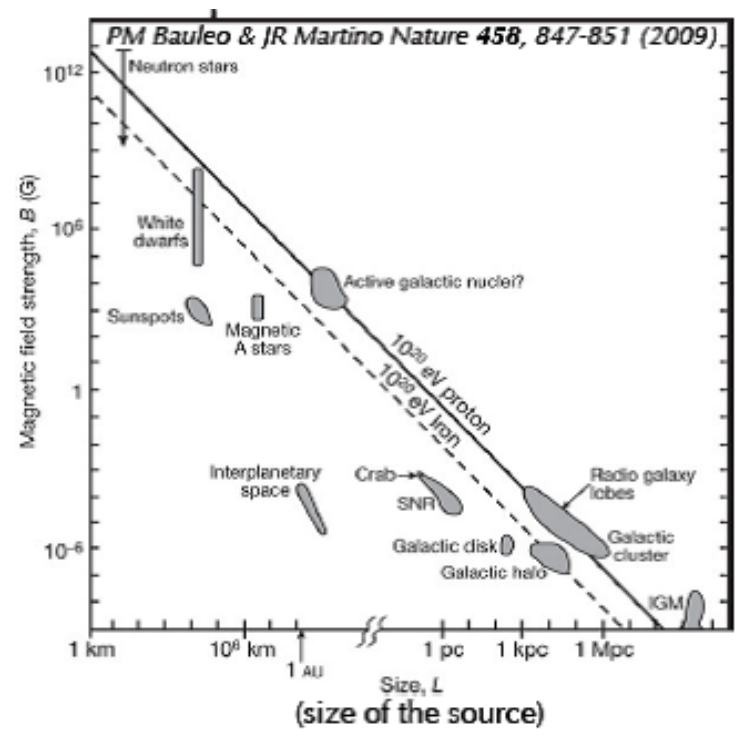

Figure 1: The Hillas Diagram. On the horizontal axis there is the typical size of the source and the vertical axis gives the typical source magnetic field. The diagonal lines correspond to the requirements for a $10^{20} \mathrm{eV}$ proton and heavy nucleus respectively. Adopted from [24].

Here, $R_{\mathrm{m}}$ represents the magnetic Reynolds number, which depends on the conductivity $(\sigma)$ of the plasma, i.e. $R_{\mathrm{m}} \propto \sigma$ (e.g. [23]). Therefore one can see that for a highly conducting fluid $(\sigma \rightarrow \infty)$, the equation for the perpendicular component reduces to the previous equation in the limit where $\Gamma \rightarrow 1$, while the component for the electric field parallel to the magnetic field $\mathbf{E}_{\|} \rightarrow 0$. However, one can see that $\mathbf{E}_{\|} \neq 0$ for a fluid with finite conductivity. This component of the electric field parallel to the magnetic field is the driving force behind field-aligned (BirkelandDessler) currents flowing in magnetospheric circuits and $\mathbf{E}_{\|} \rightarrow \infty$ for regions where $R_{\mathrm{m}} \rightarrow 0$ (i.e. $\sigma \rightarrow 0$ ), i.e. for anomalously low conductivity. If the electron conduction velocity along the field exceeds the thermal velocity $\left(v_{\text {th }}=\left(\frac{3 k T_{\mathrm{e}}}{m_{\mathrm{i}}}\right)^{1 / 2}\right)$ of the particles, microinstabilities can develop (e.g. [22]), leading to anomalously low conductivity. This can be quantified in terms of the following expression [22]

$$
\frac{B \sigma}{n_{\mathrm{e}}} \geq 10^{2}
$$

with $n_{\mathrm{e}}$ the electron density, $\sigma$ the classical plasma electrical conductivity and $B$ the magnetic field. This condition shows that microinstabilities can develop where currents flow along strong magnetic fields where the electron density $\left(n_{\mathrm{e}}\right)$ is anomalously low.

A consequence of strong field aligned currents in the magnetosphere is the acceleration of particles in strong localized field aligned potential drops, or double layers (e.g. [26, 27]). These structures can have dimensions of the order of $\sim 100-1000 \lambda_{D e}$ (e.g. [28, 29]), with $\lambda_{\mathrm{De}}=7\left(T / n_{\mathrm{e}}\right)^{1 / 2}$ representing the Debye length of electrons in a plasma. Laboratory experiments seem to indicate that multiple double layers can be induced in a system [29] and that the size of the system essentially determines the size of these structures. In regions of anomalously low conductivity the currents will reach critical values, i.e. $j_{\|}=e n_{\mathrm{e}} f c_{\mathrm{s}}$, where $c_{\mathrm{s}}$ represents the speed of sound and 
where $n_{\mathrm{e}}$ is the electron density. Here $f \rightarrow 10$ [26] represents the critical limit for the trigger of microinstabilities that will increase the resistivity of the plasma. The potential drop along the field lines can be determined from energy conservation arguments by comparing the electromagnetic Poynting flux flowing into the double layer with the particle flux out of the double layer [26], i.e.

$$
\begin{aligned}
\Phi_{\|} & =\left(\frac{\text { em flux into double layer }}{\text { particle flux out of double layer }}\right) \\
& =\left(\frac{B^{2} v_{\mathrm{a}}}{8 \pi e n_{\mathrm{e}} f c_{\mathrm{s}}}\right),
\end{aligned}
$$

where $v_{\mathrm{a}}=B / \sqrt{4 \pi \rho_{\mathrm{e}}}$ represents the Alfvén velocity. An interesting consequence of double layer formation in regions where the electron density is extremely low is that the electric field becomes uncontrollably large, resulting in an exploding double layer. These events may release a total energy reservoir $P_{\mathrm{dl}}=1 / 2 L I^{2}$ where $L$ is the total inductance of the circuit and $I \sim j d^{2} \sim e n_{\mathrm{p}} c d^{2}$ the current of relativistic particles [27] crossing the double layer of width $d$. The total power that can be released in accelerated particles and possible VHE emission is then (e.g. [27])

$$
\begin{aligned}
P_{\mathrm{dl}} & =\int \mathbf{j} \cdot \mathbf{E} d V \\
& =e n_{\mathrm{e}} c \Phi_{\|} d^{2} .
\end{aligned}
$$

Another particularly interesting mechanism for particle acceleration in magnetized fluids or plasmas occurs where magnetic fields of opposite polarity merge (e.g. [22]), resulting in a global topological change of the magnetic field. It was originally proposed that magnetic null-points can be a site of plasma heating and particle acceleration (e.g. [30, 31, 32]).

Particle acceleration in merging magnetic fields can be expressed very elegantly (e.g. [33, 27]) by considering the changing magnetic flux cutting through the orbit of a charged particle in the premerging fields, i.e.

$$
\frac{d \Phi_{\mathrm{B}}}{d t}=-\left(\frac{B \pi R_{\mathrm{L}}^{2}}{\tau_{\mathrm{m}}}\right)
$$

where $R_{\mathrm{L}}$ represents the Larmor radius of a spiralling charged particle and $\tau_{\mathrm{m}}=\left(R_{\mathrm{L}} / v_{\mathrm{m}}\right)$ represents the merging timescale. Here $v_{\mathrm{m}} \approx \alpha v_{\mathrm{a}}(\alpha \sim 0.1)$, where $v_{\mathrm{a}}$ represents the local Alfvén velocity (e.g. $[33,27])$. From Faraday's induction law the work done per unit charge (emf) in a single orbit [33] is

$$
e m f=\left(\frac{\pi B R_{\mathrm{L}} v_{\mathrm{m}}}{c}\right) .
$$

The electric field in the merging region (MR) is of the order of

$$
E_{\mathrm{MR}}=\left(\frac{\pi B v_{\mathrm{m}}}{c}\right)
$$

and must be of the same order as the field that drives a current in the dissipative region (DR), which is $E_{\mathrm{DR}}=(\mathrm{J} / \sigma)$ [34]. In order to create a strong electric field, the conductivity must be anomalously low $(\sigma \rightarrow 0)$. As a charged particle accelerates in the field, the Larmor radius will increase. The 
maximum energy that can be attained occurs if the Larmor radius becomes comparable to the width of the current sheet, which is determined by Ampére's law, i.e.

$$
w=\left(\frac{c B}{4 \pi j}\right)
$$

where $j=e n_{\mathrm{e}} v_{\mathrm{d}}$ represents the current density in the diffusive region, where the drift speed $v_{\mathrm{d}} \leq$ $f c_{\mathrm{s}}$, with $c_{\mathrm{S}}$ representing the local sound speed. The limit $f \rightarrow 10$ represents the regime where microinstabilities will be excited that will result in anomalously low conductivity in the fluid (e.g [27]). The maximum energy to which electrons can be accelerated is then (e.g. [27])

$$
\varepsilon_{\max }=\left(\frac{\pi f e B v_{\mathrm{a}} w}{c}\right)
$$

in the limit $f \rightarrow 10$. However, the real maximum energy will be determined by comparing the acceleration timescale, i.e. the time it will take the fields to merge $\tau_{\mathrm{a}}=\left(w / v_{\mathrm{m}}\right)$, with the dominant energy loss mechanisms, for example synchrotron losses $\tau_{\mathrm{s}}=2.37 \times 10^{-3} B^{2} \varepsilon_{\max }$ (e.g. [27]). It has been shown [27] that the synchrotron loss time is several orders $\left(\sim 10^{4}\right)$ longer than the acceleration timescale for a $T \sim 10^{4} \mathrm{~K}$ plasma, with an electron density of $n_{\mathrm{e}} \sim 10^{10} \mathrm{~cm}^{-3}$ in a $B \sim 1000 \mathrm{G}$ field.

The purpose of the discussion in this section was to provide a very basic framework to evaluate the non-thermal VHE emission from the enigmatic propeller driven novalike variable AE Aquarii.

\section{AE Aquarii: A Propeller Diven Pulsar}

The enigmatic multi-wavelength properties of the novalike variable star AE Aquarii have resulted in it being probably one of the best studied and documented sources in the sky. The source displays transient emission from radio wavelengths up to possibly burst-like VHE gamma-rays (e.g. $[35,36])$. The source consists of a rapidly rotating $\left(P_{*}=33 \mathrm{~s}\right)$, highly magnetized white dwarf $\left(B_{*} \sim 10^{6} \mathrm{G}\right)$ orbiting a K3-4 secondary star every $P_{\text {orb }} \sim 10 \mathrm{~h}$ (e.g. [36]). This makes the white dwarf in AE Aquarii one of the most asynchronous rotators among the cataclysmic variables [6]. A pulse timing study of the $33 \mathrm{~s}$ rotation period of AE Aquarii [37] shows that the white dwarf is spinning down at a rate of $\dot{P} \sim 5 \times 10^{-14} \mathrm{~s} \mathrm{~s}^{-1}$, implying a spin-down luminosity of the order of $P_{\text {sd }} \sim 10^{34} \mathrm{erg} \mathrm{s}^{-1}$, which exceeds the accretion power $L_{\text {acc }} \sim 10^{31} \mathrm{erg} \mathrm{s}^{-1}$ inferred from HST UV and other x-ray observations, by a factor of $\sim 1000$ (e.g. [35]). This implies that the white dwarf in $\mathrm{AE}$ Aquarii is in a propeller phase, with very little material accreting onto the surface of the white dwarf.

From a model independent energetics point it can be shown that the fast rotation period, combined with an inferred magnetic field strength of $B_{*} \sim 10^{6} \mathrm{G}$ based on circular polarization measurements (e.g. [36]), provide the required potential (e.g. see Fig. 1) to accelerate charged particles in the white dwarf magnetosphere $\left(R \sim 10^{8} \mathrm{~cm}-10^{10} \mathrm{~cm}\right)$ to energies of the order of $\varepsilon_{\max } \leq 10^{20} \mathrm{eV}$. This essentially shows that fast rotating, highly magnetized white dwarfs provide the required energetics to accelerate charged particles to the highest energies. This provides interesting possibilities for possible high energy emission.

AE Aquarii has been studied extensively in the VHE regime in the late 1980s and 1990's by two independent groups with telescopes at Nooitgedacht (South Africa) and Narrabri (Australia) 


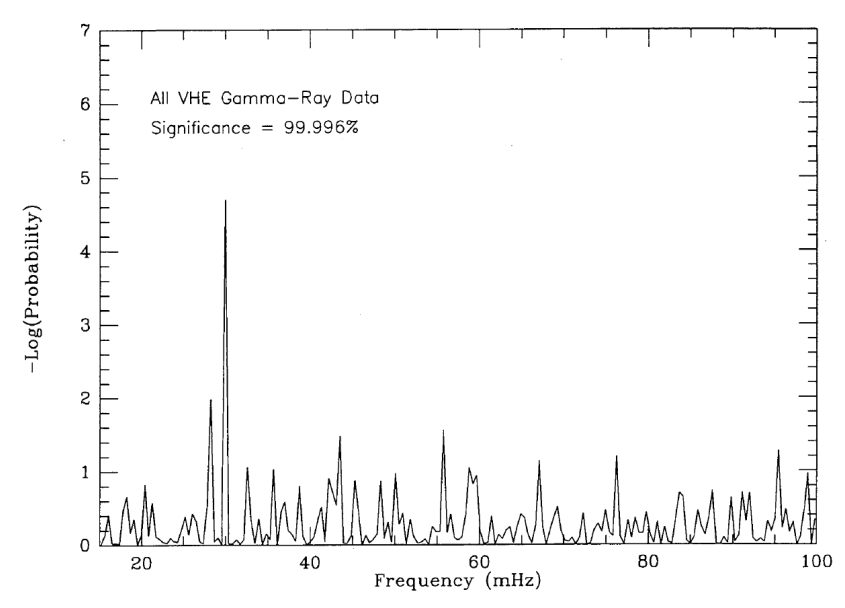

Figure 2: Pulsed $\mathrm{TeV}$ gamma-ray emission from $\mathrm{AE}$ Aquarii at a period close to the spin period of the white dwarf utilizing the total data set spanning 4 years. Adopted from [17].

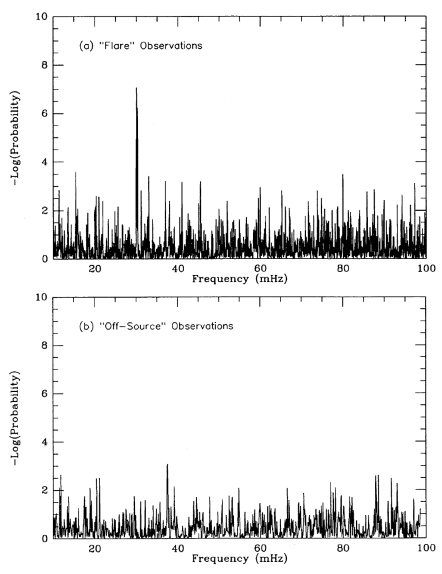

Figure 3: Pulsed TeV gammaray emission from AE Aquarii during periods of enhanced activity. Adopted from [17].

respectively (e.g. [16, 17, 18]). Between 1988 and 1991 the Nooitgedacht Cerenkov array has observed AE Aquarii for $\sim 240$ hours on moonless nights and registered $\sim 233600$ Cerenkov events [17]. By utilizing the complete dataset spanning 4 years, a pulsed modulation close to the white dwarf spin period of $33 \mathrm{~s}$ has been detected at the $99.996 \%$ significance level (e.g. Fig. 2).

Simultaneous, or contemporaneous optical observations performed utilizing telescopes at the South African Astronomical Observatory (SAAO) that showed flaring activity were used to extract the VHE gamma-ray observations where the source was active. These observations were analysed for periodicity and compared to comparison "off-source" observations at the same nights. This showed that the pulsed VHE emission at $33 \mathrm{~s}$ is associated with periods of enhanced activity of the source, with no modulation at the same period detected in the comparison off-source data (Fig. 3).

Apart from this consistent pulsed emission close to the spin period of the white dwarf, burst like emission (e.g. Fig. 4 and Fig 5) have been detected by both VHE gamma-ray groups [16, 18]. During the night of the Nooitgedacht burst, simultaneous optical observations were made utilizing the $0.75 \mathrm{~m}$ telescope at SAAO. It can be seen that the source showed significant optical variability during that particular night. The bottom panel of Fig 4 shows the photocurrents of the three operational Nooitgedacht Cerenkov telescopes to confirm that the TeV burst was not the result of instrumental instability. Both these burst showed double peaked pulsed emission when folded with the $P_{*}=33.08 \mathrm{~s}$ white dwarf period, implying that both poles of the rotating white dwarf is visible in VHE gamma-rays $[16,18]$.

The nature of the non-thermal emission from AE Aquarii, from radio to VHE gamma-ray gamma-rays, have been investigated in detail by [38], [27] and [39]. It has been shown that episodes of particle acceleration may be associated with the propeller process in AE Aquarii. Magnetic shear in the ejection region will induce field-aligned currents in the magnetosphere. Huge potential differences (double layers) can be generated in the circuit where the gas density becomes very low. This will occur close to the white dwarf. In this region potentials of the order of $\Phi \sim 300 \mathrm{TV}$ (TV $=$ teravolt) can be generated. Synchrotron losses on electrons close to the white dwarf will restrict 

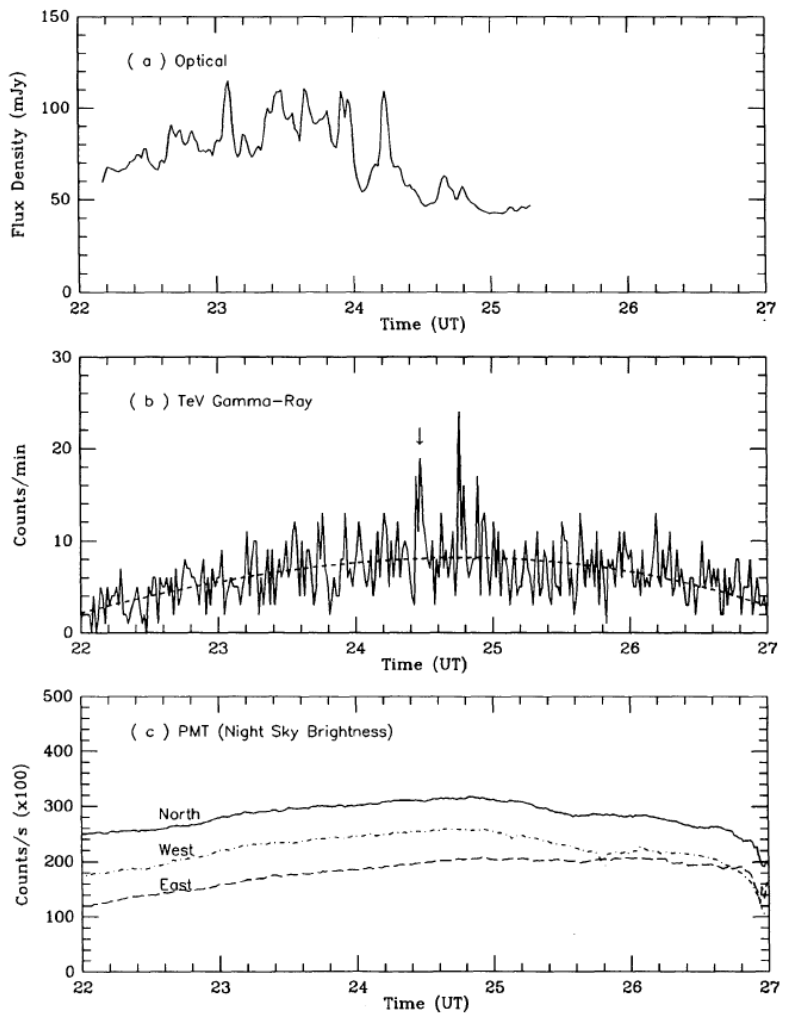

Figure 4: Burst-like TeV gamma-ray emission from AE Aquarii observed with the Nooitgedacht Cerenkov telescope in South Africa. Adopted from [18].
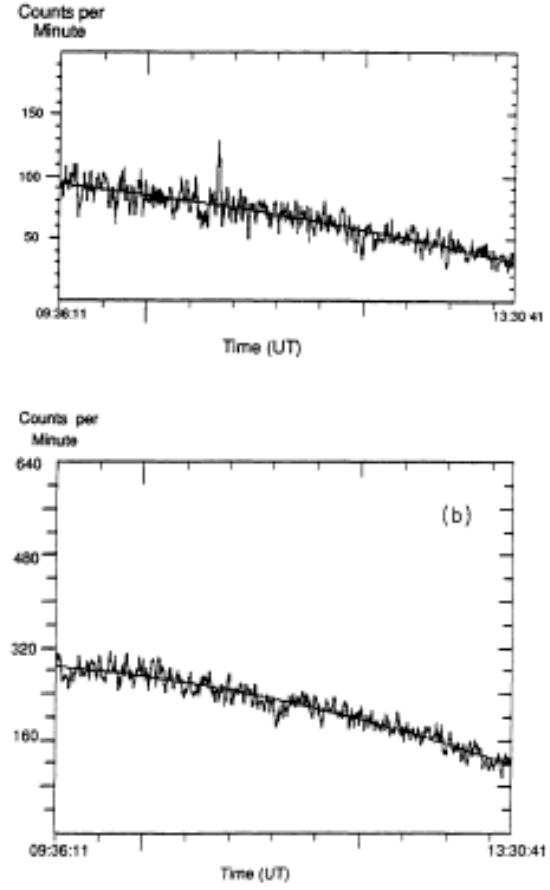

Figure 5: Burst-like VHE gammaray emission from $\mathrm{AE}$ Aquarii observed with the Cerenkov telescope in Narrabri. Adopted from [16].

them to energies of the order of $\mathrm{E}<50 \mathrm{keV}$. As protons and ions are not affected by synchrotron losses, energies in excess of $1 \mathrm{TeV}$ are possible. Very high-energy (VHE) gamma-ray emission can be produced if these proton and ion beams collide with the clumpy gas in, or outside, the ejection zone. It has been shown that pulsed VHE gamma-ray emission is possible, if the gas particle density of the target matter is approximately $N_{\mathrm{g}} \sim 10^{13} \mathrm{~cm}^{-3}$, which is compatible with the typical gas particle densities $N_{\mathrm{g}} \sim 10^{16} \mathrm{~cm}^{-3}$ of the blobs inside the clumpy accretion stream from the secondary star. We show that the VHE gamma-ray bursts that were reported by the two groups can be explained in terms of an exploding double layer, which will result in a cataclysmic burst of VHE protons and ions over short time-scales. The total power released in these bursts can be $P_{b} \sim 10^{34} \mathrm{erg} \mathrm{s}^{-1}$, which is equal to the spin-down power of the white dwarf, and is enough to explain the reported VHE gamma-ray bursts. This implies that the whole reservoir of spin-down power has been utilized for the production of VHE particles and gamma-ray emission.

More recently a study of the properties of the hard X-ray $(\varepsilon>10 \mathrm{keV})$ pulsations of $\mathrm{AE}$ Aquarii detected in the Suzaku data [40], revealed that AE Aquarii displays the same ratio $\left(\sim 10^{-4}\right)$ of hard X-ray luminosity $\left(L_{\mathrm{X}} \sim 10^{30} \mathrm{erg} \mathrm{s}^{-1}\right)$ to spin-down power $\left(P_{\mathrm{sd}} \sim 10^{34} \mathrm{erg} \mathrm{s}^{-1}\right)$, which is similar to the relationship normally observed for young spin-powered pulsars [41]. Furthermore, the Suzaku hard X-ray spectrum seems to suggest a power-law shape, with a photon index of $\alpha \sim 2.3$ [40], indicative of a non-thermal emission process. The pulsar-like acceleration of particles 


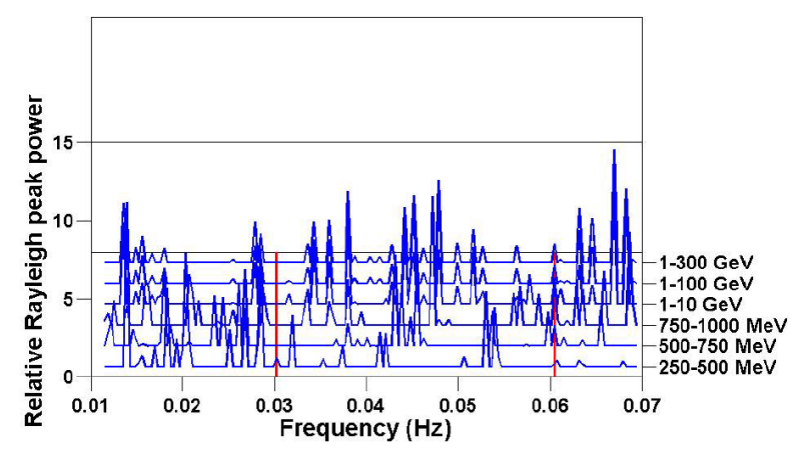

Figure 6: A search for pulsed emission of AE Aquarii in various energy bins of the Fermi-LAT data. Notice the presence of weak pulsed modulation in all bins simultanously. Adopted from [42].

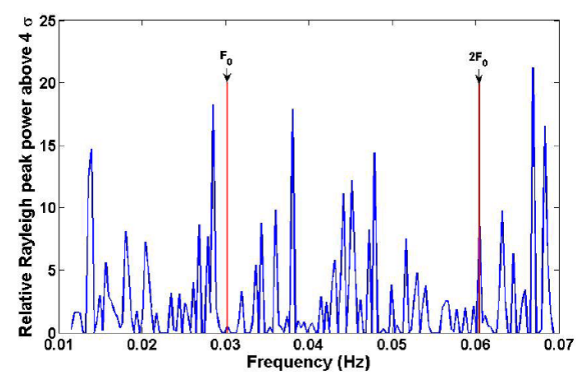

Figure 7: Combined power spectrum revealing weak pulsations at $2 F_{\circ}(16.53 \mathrm{~s})$, which is just the first overtone of the fundamental spin period. Adopted from [42].

in the white dwarf magnetosphere in AE Aquarii has been investigated by [35] and [39]. It has been shown that huge potentials of the order of $V \sim 3 \times 10^{12} P_{33}^{-2} \mu_{33}$ Volt can exist between the white dwarf surface and the light cylinder, which can accelerate charged particles to VHE-TeV energies. Here $P_{33}$ and $\mu_{33}$ represent the white dwarf spin period (33 s) and the magnetic moment of the white dwarf $\left(\sim 10^{33} \mathrm{G} \mathrm{cm}^{3}\right)$ respectively. Keeping in mind synchrotron losses, these authors showed that relativistic electrons with Lorentz factors of the order of $\gamma \sim 10^{5}$ can be produced inside the light cylinder of the white dwarf, and will radiate synchrotron radiation in the magnetospheric field $\left(B_{\mathrm{lc}} \sim 100 \mathrm{G}\right)$ in the proximity of the light cylinder at frequencies $v \sim$ few $\times 10^{18} B_{100} \gamma_{5}^{2} \mathrm{~Hz}$, where $B_{100}$ and $\gamma_{5}$ represent the magnetic field $(\sim 100 \mathrm{G})$ in the proximity of the light cylinder and Lorentz factor $\left(\sim 10^{5}\right)$ respectively. It was further shown that the inverse-Compton scattering of $\gamma \sim 10^{5}$ electrons on optical photons from the secondary star $\left(\varepsilon_{\mathrm{ph}}<5 \mathrm{eV}\right)$, provide interesting possibilities for the production of gamma-rays with energies of the order $\varepsilon_{\gamma} \leq 0.2 \gamma_{5}^{2}\left(\varepsilon_{\mathrm{ph}} / 5 \mathrm{eV}\right) \mathrm{TeV}$, which would place it in the Fermi-LAT energy range [35, 39].

As part of a $\mathrm{PhD}$ study a search for pulsed non-thermal emission was conducted utlizing the Suzaku, AGILE and Fermi-LAT data archives [42]. This study revealed no steady emission above the noise-level, but some weak consistent pulsed signatures at the first harmonic $2 F_{\circ}$ ( $16.54 \mathrm{~s}$ ) of the fundamental frequency $F_{\circ}(33.08 \mathrm{~s})$ in the Fermi-LAT data. In a search, involving separate energy bins, it has been shown [42] that a consistent modulation at $2 F_{\circ}(=16.64 \mathrm{~s})$ is present in all the energy bins (see Fig. 6). The power spectrum involving the combination of the energy bins reveals a consistent pulsation at $2 F_{\circ}$, albeit in the noise level regarding overall significance (e.g. Fig. 7). If these pulsations are real, it could be interpreted possibly in terms of the pulsar-like emission from both polar caps of the fast rotating white dwarf pulsar in the AE Aquarii system. No significant emission has been detected at the $F_{\circ}(33.08 \mathrm{~s})$ fundamental frequency.

\section{Discussion}

In this paper an overview is presented of some processes driving particle acceleration and nonthermal transient emission processes in close binaries. It has specifically been applied to explain the reported non-thermal emission in AE Aquarii. It has been shown that strong field aligned 
potentials in the white dwarf magnetosphere can accelerate protons to VHE energies. The burstlike VHE-TeV gamma-ray emission, reported from two groups independently in the 1990s, can be explained readily in terms of exploding double layers that can release instantanously the full reservoir of spin-down energy of the white dwarf in relativistic protons and subsequent gamma-ray emission through $\pi^{\circ}$ decay. A recent study that focussed on the search for pulsed emission from AE Aquarii in the Fermi-LAT data revealed weak but consistent pulsed signatures in the noise, at a period of $16.54 \mathrm{~s}$, which is just the first overtone of the fundamental spin period of the white dwarf.

\section{Acknowledgements}

The author appreciates the invitation from the organising committee to present these results at the conference and for the hospitality during the conference.

\section{References}

[1] M. Cropper, The Polars, Space Science Reviews 54 (1990) 195

[2] W.H.G. Lewin, J. van Paradijs \& P.J. van den Heuvel, X-Ray Binaries, Cambridge Astrophysics Series, 26 (1995)

[3] N.U. Shakura \& R.A. Sunyaev, Black holes in binary systems. Observational appearance, $A \& A 24$ (1997) 337

[4] F. Meyer \& E. Meyer-Hofmeister, On the Elusive Cause of Cataclysmic Variable Outbursts, A\&A 104 (1981) L10

[5] V.M. Lipunov, Astrophysics of Neutron Stars, Astronomy and Astrophysics Library, SpringerVerlag (1992)

[6] J. Patterson, The DQ Herculis Stars, PASP 106 (1994) 209

[7] G.A. Wynn \& A.R. King, Diamagnetic accretion in intermediate polars - I Blob orbits and spin evolution, MNRAS 275 (1995) 9

[8] G.A. Wynn, A.R. King \& K. Horne, A magnetic propeller in the cataclysmic variable AE Aquarii, MNRAS 286 (1997) 436

[9] P.J. Meintjes \& L.A. Venter, The diamagnetic blob propeller in AE Aquarii and the nonthermal radio to mid-infrared emission, MNRAS 360 (2005) 573

[10] D.V. Bisikalo \& A.G. Zhilkin, Accretion processes in intermediate polars with asynchronous rotations of white dwarfs, in proceedings of Golden Age of Cataclysmic Variables and Related Objects, Palermo, (eds. F. Giovanelli \& L. Sabau-Graziati), MEMORIE S.A.It 83 (2) (2012) 562

[11] G. Chanmugam \& G.A. Dulk, AM Herculis Binaries: Particle Acceleration, Radio Emission and Synchronization, in Proceedings of Cataclysmic Variables and Related Objects (eds. M. Livio and G. Shaviv) Dordrecht Reidel Publishing Company (1983) 223 
[12] G.A. Dulk, T.A. Bastian \& G. Chanmugam, Radio Emission from AM Herculis: The quiescent component and outburst, ApJ 273 (1983) 249

[13] J.A. Bookbinder \& D.Q. Lamb, Discovery of radio emission fro AE Aquarii, ApJ 323 (1987) L131

[14] T.S. Bastian, G.A. Dulk \& G. Chanmugam, Radio Flares from AE Aquarii: A low power analogue to Cyg X-3, ApJ 324 (1988) 431

[15] P.A. Mason \& C. Gray, An investigation of radio emission in magnetic cataclysmic variables, in the Proceedings of IAU Colloquium 190: Magnetic Cataclysmic Variables, ASP Conf. Series, 315 (2004) (eds. S. Vrielman \& M. Cropper), San Francisco, 237

[16] C.C.G. Bowden et al., $350 \mathrm{GeV}$ gamma-rays from AE Aquarii, Astropart. Phys. 1 (1992) 47

[17] P.J. Meintjes et al., AE Aquarii: An emitter of pulsed TeV gamma-rays resembling optical emission during flares, ApJ 401 (1992) 325

[18] P.J. Meintjes et al., Simultaneous optical and TeV gamma-ray observations of the cataclysmic variable AE Aquarii, ApJ 434 (1994) 292

[19] M.J. Lang et al. A search for TeV gamma-ray emission from AE Aquarii, Astropart. Phys. 9 (1998) 203

[20] C.W. Mauche et al., Multiwavelength campaign of observations of AE Aquarii, in Proceedings of Golden Age of Cataclysmic variables and Related Objects (eds. F. Giovanelli \& L. SabauGraziati), Mem. S.A. It Vol 83 (2) (2012) 651

[21] R. López-Coto et al.. A search for TeV $\gamma$-ray emission from AE Aquarii coincident with high optical and X-ray states with the MAGIC telescopes, in Proceedings of The 33 rd international cosmic-ray conference, Rio de Janeiro 2013 arXiv:1309.2503v1 [astrp-ph.HE] 10 Sep 2103

[22] E.N. Parker, The acceleration of particles to high energy, in proceedings of The Physics of Non-Thermal Radio Sources (ed. G.Setti), Dordrecht-Reidel Publishing Company, (1976) 137

[23] J.D. Jackson, Classical Electrodynamics (2nd ed), Wiley \& Sons, New York, (1975) 473, 552

[24] P.M. Bauleo \& J.R. Martino, The dawn of the particle astronomy era in ultra-high energy cosmic sources, Nature $\mathbf{4 5 8}$ (2009) 847

[25] L. Mestel Stellar Magnetism, Claredon Press Oxford, (1999) 533- 630

[26] G. Haerendel, Particle acceleration from field aligned potential droms, ApJS (1994) 90765

[27] P.J. Meintjes \& O.C. de Jager, Propeller spin-down and the non-thermal emission from AE Aquarii, MNRAS 311 (2000), 611

[28] M.A. Raadu, The physics of double layers and their role in astrophysics, Phys. Reports $\mathbf{1 7 8}$ (1989) 25 
[29] M.A. Raadu, Energy release in double layers, Space Science Reviews 68 (1994) 29

[30] R.G. Giovanelli, A theory of chromospheric flares, Nature (1946) 15881

[31] F. Hoyle, Some recent advances in Solar Physics, Cambridge Univ. Press, Cambridge (1949)

[32] E. Priest \& T. Forbes, Magnetic Reconnection: MHD theory and application Cambridge Univ. Press, Cambridge (2000) pp. 1-45

[33] D. Biskamp, Hot spots in extragalactic radio sources, Lecture Notes in Physics (eds. K. Meisenheimer \& H.J Röser), Springer Berlin (1988) 279

[34] A. Benz, Plasma Astrophysics, Astrophys. Space Science Library 184 Kluwer Academic Publishers, Dordrecht (1994) 285

[35] P.J. Meintjes, B Oruru \& A. Odendaal, The multi-frequency properties of AE Aquarii and its evolution form a high mass transfer history, in Proceedings of Golden Age of Cataclysmic Variables and related Objects (eds. F. Giovanelli \& L. Sabau-Graziati), Mem. S.A.It. 83 (2012) 643

[36] P.J. Meintjes, A Odendaal \& H.J. van Heerden, AE Aquarii: A short review in Proceedings of Golden Age of Cataclysmic Variables and related Objects II (eds. F. Giovanelli \& L. Sabau-Graziati) Acta Polytechnica CTU proceedings 2(1) (2015) 86

[37] O.C. de Jager, P.J. Meintjes, D. O'Donoghue \& E. Robinson, The discovery of a brake on the white dwarf in AE Aquarii, MNRAS 267 (1994) 577

[38] O.C. de Jager, Evidence for particle acceleration in a magnetized white dwarf from radio to gamma-ray observations ApJ Suppl. 90 (1994) 775

[39] B. Oruru \& P.J. Meintjes, X-ray characteristics and the spectral energy distribution of $A E$ Aquarii, MNRAS 421 (2012) 1557

[40] Y. Terada et al., Suzaku discovery of hard X-ray pulsations from a rotating magnetized white dwarf, AE Aquarii, Publ. Astron. Soc. Japan 60 (2008) 387

[41] W. Becker \& J. Trümper, The x-ray luminosity of rotation powered neutron stars, A\&A $\mathbf{3 2 6}$ (1997) 682

[42] H.J. van Heerden, A search for pulsed high-energy non-thermal emission from the nova-like variable system AE Aquarii, PhD-Thesis (2015), Univ. of the Free State (unpublished) 